\title{
Evaluation of Idle Speed Control Method of Automotive Engine
}

\author{
Sun Jianmin ${ }^{1, a}$, Yin Fengfeng ${ }^{2, b}$ and Wei Zhiyuan ${ }^{3, C}$
}

${ }^{1}$ School of Mechanical-electronic and Automobile Engineering, Beijing University of Civil
Engineering and Architecture, 100044,China

\author{
${ }^{2}$ School of Mechanical-electronic and Automobile Engineering, Beijing University of Civil \\ Engineering and Architecture, 100044,China \\ ${ }^{3}$ School of Mechanical-electronic and Automobile Engineering, Beijing University of Civil \\ Engineering and Architecture, 100044,China \\ aemail: jinminsun@tum.com, bemail: m17888812565@163.com, email: 15901127824@163.com
}

Keywords: Engine; Idle speed system, Control algorithm

\begin{abstract}
Automotive Idle speed control is a landmark application of feedback control in automotive vehicles that continues to be of significant interest to automotive industry practitioners, since improved idle performance and robustness translate into better fuel economy, emissions and drivability. As the typical automotive transient operation, the quality of the ISC has a significant impact on fuel economy, emission levels, combustion stability, transient response, and noise, vibration, and harshness characteristics. This paper reviews the current various working condition of the engine idle speed control method, including the now widespread use of classical control, modern control and intelligent control.
\end{abstract}

\section{Introduction}

The engine idle speed conditions is in foreign under the condition of not doing any work, with the lowest stable speed running state.Completely engine and drive train from, its purpose is to maintain the engine at low speed under the continuous, stable operation and provide other various auxiliary device of work motivation, such as air conditioning, power steering suddenly open or closed, the engine speed and stable operation at a certain speed range.The major factors influencing the performances of the engine idle speed are two aspects. One is to control the flow of mixed gas into the cylinder.Because the mixture flow directly affect the mixture in the combustion chamber combustion speed, pressure and temperature, thereby the engine power performance, fuel economy and exhaust pollutant composition has a great influence.On the other hand is to the mixture in cylinder ignition moment, different ignition moment also can produce very big effect to the process of the combustion in cylinder, which affects the dynamic performance of engine.

\section{Engine Idle Speed Control Strategy}

In idle speed control problem due to its specific goals, which in the case of uncertain interference, ensuring the engine speed to the reference speed good steady-state performance and dynamic performance can be a very typical closed-loop feedback control problem.Engine idle speed control scheme as shown in Fig.1.Many researchers abroad on the basis of the basic control strategy, joined the ignition moment control [1-3].Adopting the method of changing the ignition advance angle to control working condition of stable idle speed of response is much faster and can achieve higher precision, but changing the ignition advance angle can tinily change the speed range.Domestic researchers have carried out the research work.In [4,5],they investigated different engine model and control method in idle speed control applications,and discusse the control system of linear motor Mexico's analysis and synthesis and simulation and controller design of nonlinear system.Compared with the traditional control method and the advantages and disadvantages of the advanced control method.So far, many advanced control theory has been applied to the problem of idle speed control in [6], such as fuzzy control, adaptive control and robust control method.Summarize the engine idle 
speed aspects of the related literature at home and abroad,the following are the main types of engine idle speed control.

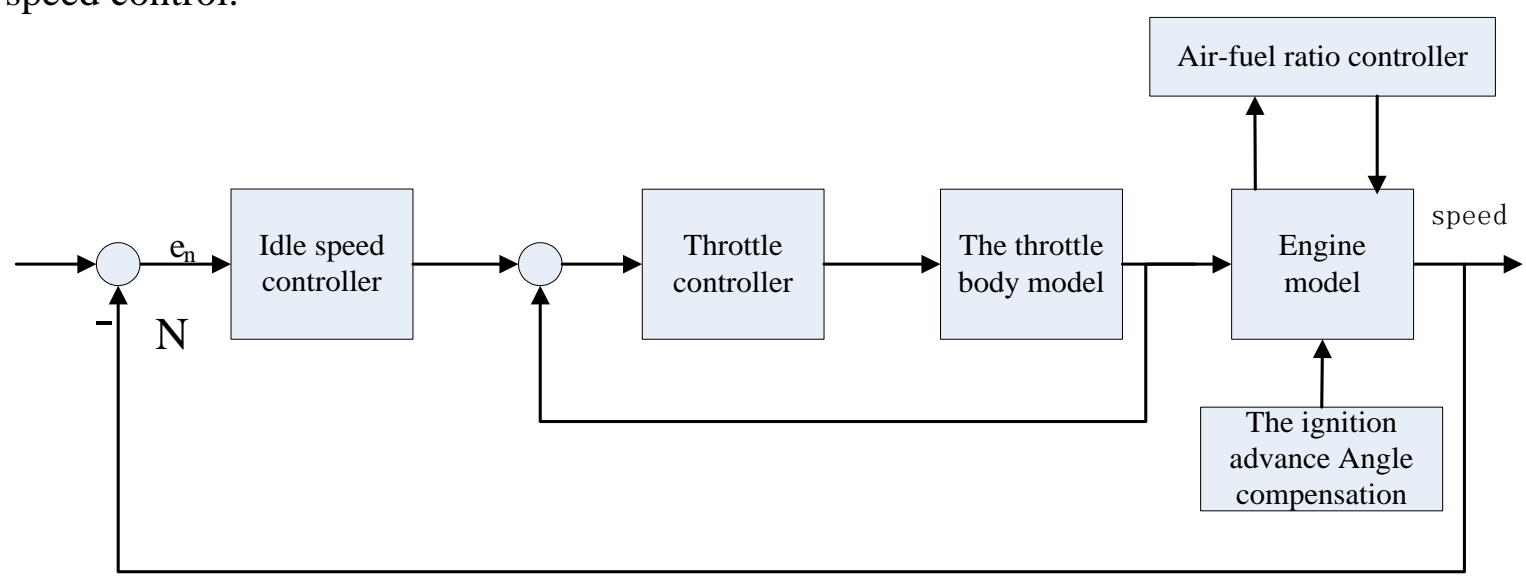

Fig. 1 Engine idle speed control scheme

\subsection{The Idle Neural Network Control}

Neural network has been widely used in the engine idle speed control system modeling, the observer design and the design of the controller. When the working condition of automobile engine at idle speed, inlet pressure, air-fuel ratio, ignition time, moving parts and friction factor will affect the stability of idle speed.A combination of these factors make idle process is a complicated system, it is difficult to use mathematical model to accurate description, so many researchers tend to use the fuzzy control and neural network control method.Neural network has infinite ability to approximate nonlinear function, it has the parallel processing, distributed storage, fault tolerance and adaptive ability.But the commonly used BP algorithm belongs to the global approximation network, slow learning speed,it is difficult to meet the real-time requirements of automobile engine.Engine idle speed neural network control system block Fig.2.

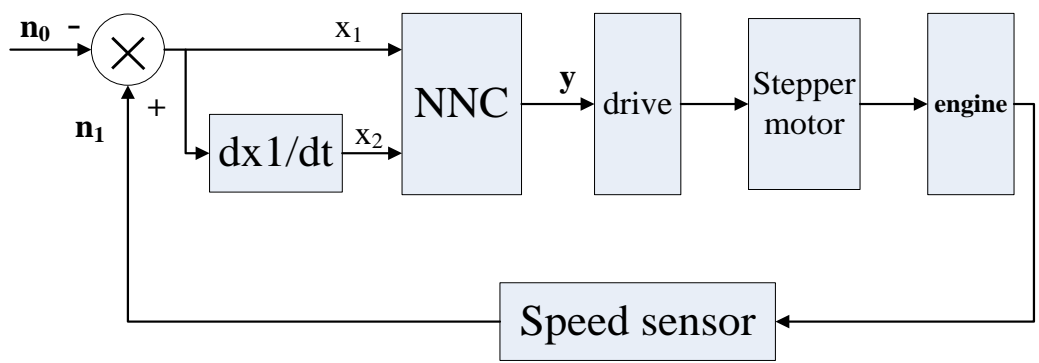

Fig. 2 Engine Idle Speed Neural Network Control System Block Diagram

In [7],it combined neural network and fuzzy control to control transmitter idle condition, solves the engine time-varying, nonlinear and model uncertainty.Through the simulation, the dynamic performance of engine idle speed system has greatly improved and the change of model parameters has good robustness, due to the map input and output has nonlinear relationship in idle speed control, leading to step feature.In[8],it utilize the nonlinear mapping ability of RBF neural network and the gradient descent method with forgetting factor is applied to RBF neural network parameter adjustment.Based on engine speed and speed rate mapping, getting stepper motor drive signal, so as to realize the idle speed control.The experimental results show that in this kind of condition by using RBF neural network control is a very effective strategy, control of fast response, strong robustness, which can effectively reduce the engine speed fluctuation, further improve the stability of the engine idle speed running.In[9], idle speed control is realized by using the BP neural network method, through comparing with the traditional PID control method, it shows that the BP neural network control has better performance and it is a very effective control strategy can effectively reduce the engine speed fluctuations, to further improve the stability of the engine idle speed running.

\subsection{The Idle Model Predicts}

Through online optimization and model predictive control loop feedback correction in the implementation of control function and model error,it can effectively overcome the system 
uncertainties and nonlinear, it has good robustness.Current predictive control algorithm of 3 typical model algorithm control, dynamic matrix control and generalized predictive control in the engine idle speed control system of preliminary application, but they exist the common problem is that the huge computational overhead, it is difficult to ensure the real-time demand of the algorithm with embedded microcontroller controller as the carrier of the operation unit.Engine idle speed control prediction block diagram as shown in Fig. 3.

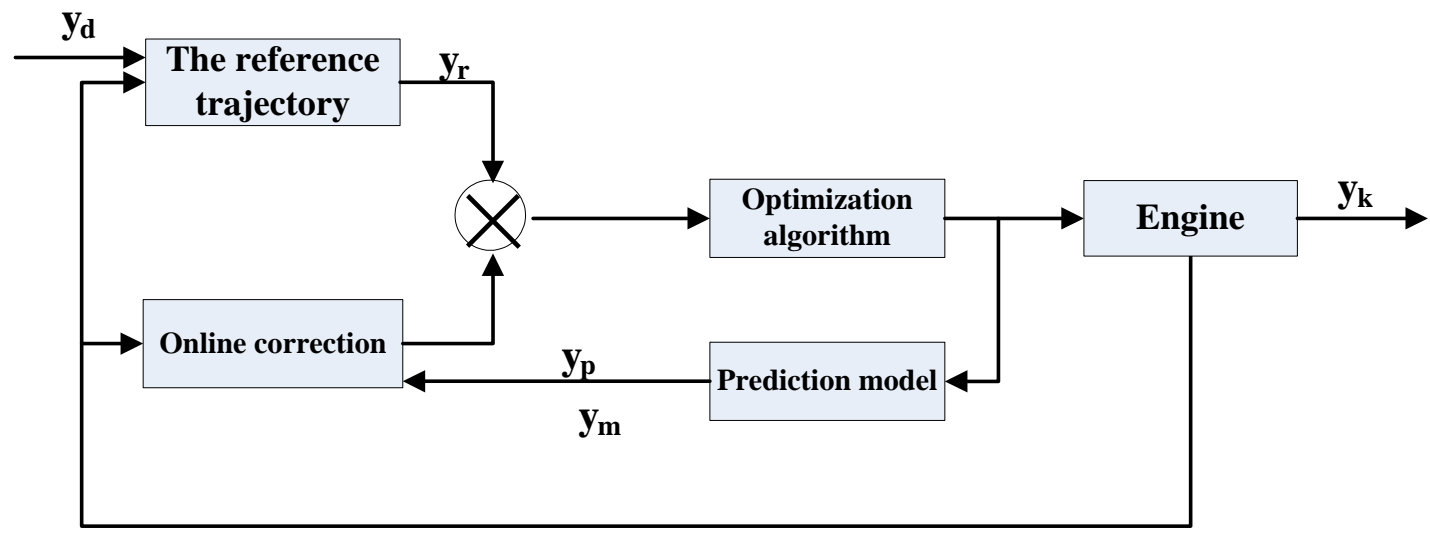

Fig. 3 Engine idle speed control prediction block diagram

In [10],it use the method of model predictive control to control the air inflow of engine idle speed and ignition timing. Through test and actual vehicle model predictive controller ,proved that it has good robustness, better than other control methods. In [11],it design of gasoline engine idle speed dynamic matrix predictive control (DMC) system, using the established model of engine idle speed control and DMC algorithm of engine idle speed control, idle speed of the step response model is established for the control algorithm and system parameter design to provide important basis. The results showed that the DMC algorithm is suitable for the engine idle speed control, and has good stability, strong anti-interference, etc.

\subsection{Idle Sliding Mode Control}

Neural network has the ability to learn and the ability to approximate arbitrary nonlinear function; Variable structure control can adapt to system parameter variations and external disturbances, the design method of nonlinear system is better.Gasoline engine idle speed is a nonlinear, large time delay and disturbance of the system, so in [12], it use the sliding mode control method based on neural network control idle speed of engine and the engine model is average model.it adopts the RBF neural network, neural network is used to target switching surface error, the upper boundary compensation algorithm using adaptive filtering algorithm and controller design.Simulation shows that under the steady state rotational speed fluctuation amplitude reached $10 \mathrm{r} / \mathrm{min}$, when the load mutation of droop up to $50 \mathrm{r} / \mathrm{min}$, the article did not specify the simulation forms and the environment, at the same time using single type of load, the maximum of $20 \mathrm{Nm}$.Automobile engine target idle speed value after the change, it did not give the simulation results whether controller can adapt.In [13], with the method of discrete integral sliding mode control, as a result of the sliding mode control sliding mode has nothing to do with object parameter and disturbance, this kind of model is not suitable for the engine idle speed control precision and disturbance in the larger system.Therefore sliding mode control and all kinds of advanced control methods in the engine idle speed control system must be applied. Sliding mode control weakness is that the system can produce jitter, actual idle speed control system of actuators (generally) for the throttle response speed don't meet the requirement of theory, limit the synovial control in the practical application of throttle control system.

\subsection{H $\infty$ Control Theory}

$\mathrm{H} \infty$ controller also is less in the study of idle speed control, $\mathrm{H} \infty$ control of model parameter uncertainty and external torque has strong robustness, it will make its development step.But for large interference, when using the multi-objective control h-infinity controller design,it can not completely eliminate the influence of disturbance.At this point, there are usually two methods combined with h-infinity controller to improve the system's ability to restrain disturbance, one is the rolling 
optimization strategy, through continuous optimization controller to achieve full of interference suppression, the dynamic response process of this method has strong shock; the other one is disturbance compensation control, and disturbance observer in the disturbance compensation control is a common, it estimates based on the interference to offset in the control input to interference.

In [14], the Hळo control theory for the engine idle speed control, the establishment of a LPG engine idle speed control object model, based on the model to solve the sensitivity of the function and the complementary sensitivity function, it mixed sensitivity problem into a standard $\mathrm{H} \infty$ control problem, the simulation and test results show that the $\mathrm{H} \infty$ control control has better robust stability compared with PI, improve the stability of idle speed condition.In[15], in view of the uncertainty of CNG engine, the $\mathrm{H} \infty$ robust control theory is applied to $\mathrm{CNG}$ engine idle speed control, this paper expounds the $\mathrm{H} \infty$ weighted mixed sensitivity design model, to establish the dynamics model of CNG engine, the uncertainty of the engine are analyzed.Application Matlab robust control toolbox designed a $\mathrm{CNG}$ engine idle speed based on the theory of the $\mathrm{H} \infty$ controller and it has carried on the simulation analysis and experiment.The results show that the $\mathrm{H} \infty$ control has good robustness, improve the stability of CNG engine idle speed condition.In[16],it based on Linear matrix inequality (LMI) LMI (Linear matrix inequal-ity, LMI) robust control method to design the $\mathrm{H} \infty$ constrained idle speed controller, the simulation experiments show that the controller to throttle time-domain hard constraints have very good control effect and the parameter uncertainty has very strong robustness.

\section{The Development Trend of Engine Idle Speed Control System}

In conclusion,the car engine idle speed control technology has made remarkable progress, however, the dynamic mechanism are complex in the car engine idle speed control system, performance index interact with each other, a large number of new technology is widely used at the same time,it meets the automobile power performance, safety and energy conservation and emissions reduction, but it introduces many new type electric actuators, adds the control degrees of freedom and the complexity of the dynamic coupling and made the electric control system design, calibration and test more difficult.It puts forward a new challenge to the traditional control theory and method of application.In short, there were many important and unsolved problems need to be engaged in auto control researchers to explore and cognition in car engine idle speed control, the following summarizes some common problems to solve these problems and puts forward some possible development trend of automobile engine idle speed control system.

\subsection{Idle Speed Control Valve is Introduced Into The Safety Monitoring}

By-pass air control idle speed control mechanism is known as the idle speed control valve (ISC valve).In the actual system, air by-pass valve's structure and control mode are different,it generally can be divided into: stepper motor regulating mechanism, duty ratio control type solenoid valve, switch control type solenoid valve [17].Idle speed control of a proven effective way is through the bypass valve to control the air flow, now practice also have to adjust the throttle idle speed limiter or use engine electronic power control system of direct drive throttle [18].Now auto idle speed control system introduced the function of security monitoring, idle speed control system of gas input by the electronic throttle control, if there is an error signal, software or hardware errors occur, electronic throttle could spiral out of control, any one of these conditions occur will lead to the car out of control and may cause dangerous situation.But the engine idle speed control system is introduced into the function of security monitoring can effectively avoid the risk of similar.Therefore, the car idle speed control system is introduced into safety monitoring capability is a development direction in the future.

\subsection{Engine Calibration, Match, and The Simulation Platform Development}

Engine calibration/matching process need to repeatedly adjust the control parameters to the experimenter, and repeatedly adjust MAP figure according to the results of the experiment,working process is very complicated, and with the improvement of engine control system mixed degree, more and more control parameters need to optimize, engine calibration/matching process is also present a trend of more and more complex.Auto control of simulation research mostly stay in the functional design and validation phase, the offline simulation is often a simple controller and some real vehicle test is only carried out under normal conditions. In order to more fully demonstrate the effectiveness 
of the controller, however, some of the real vehicle test shall be carried out under the extremely harsh environment, with a certain risk, at the same time, the control algorithm for real vehicle need for real-time validation. Therefore, the development with the computer as the core engine electronic control system of automatic calibration/matching platform will greatly improve the efficiency of engine calibration/match;Build hardware in the loop simulation platform which can reflect all kinds of vehicle real-time running status of high fidelity is to verify the essential means to auto control method for real-time and reliability.

\section{Conclusion}

Road motor vehicles growth rate is $15 \%$ in China in recent years the average annual and has a rising trend.This will cause more and more serious pollution. The primary cause is behind the engine control equipment.Idle speed control of the main reason for the inefficiency causes such serious pollution.Due to the special nature of idle speed condition,it maintains running and auxiliary device of the engine itself to provide energy.Therefore, in order to try to reduce fuel consumption, requirements reduce the engine speed as much as possible.At home and abroad in this paper, some of the car engine idle speed control strategies are reviewed, the choice of idle speed control method in all aspects of the idle speed control performance plays an important role, such as the stability of idle speed, fuel economy and emissions, etc.Although through the study of literature found that auto control technology has made great progress in these years, but it still exists many problems unsolved in auto control, also need to engage in auto control the direction of the scientific researchers to explore and cognition, through continuous summary and exploration,it will have a better car idle control strategy for the benefit of humanity.

\section{References}

[1]Duksun Shim,Jaehong Park. Reducing Automotive Engine Speed Fluctuation at idle[J]. IEEE. Transactions on control system technology. 1996, 4(4):2-4.

[2] Kyung-Jinn Yang, Jae Weon Choi, Keum-Shik Hong. Advanced Sliding Mode Idle Speed Control for a Nonlinear Engine Model[J]. Coordinated Throttle/Spark Advance Control. PR0001-3.SICE. 1997, 7:29-31.

[3] Christian Bohn, Thomas Bohme. A Nonlinear Model for Design and Simulation of Automotive Idle Speed Control Strategies[C].Proceeding of The 2006 American Control Conference. 2006:8-10.

[4]D.Hrovat, Jing Sun.Models and Control Methodologies For IC Engine Idle Speed Control Design[J]. Control Engineering Practice. 1997:1093-1100.

[5]Stelios Karagiorgis,Keith Glover and Nick Collings.Control Challenges in Automotive Engine Management[J].European Journal of Control (2007)13:92-104.

[6] Zhengmao Ye. Modeling,Identification,Design and Implementation of Nonlinear AutomotiveIdle Speed Control Systems-An Overview[J].IEEE TRANSACTIONS ON SYSTEMS, MAN, AND CYBERNETICS.2007: 1137〜1151.

[7]Zhao Guangzhou,Yang Zhijia. Application of the neural network fuzzy controller of engine idle speed control [J]. Journal of internal combustion engine engineering. 2000:59-62.

[8] Zhang Jinlong, Zhao Fusheng. Electronic-controlled engine idle speed RBF neural network control [J]. Journal of agricultural machinery, 2005, 4 (8) : 20 to 22 .

[9]Jian Lei Cao,Jia Meng Yin,Ji Sun Shin,Hee Hyol Lee. BP Network Modified by Particle Swarm Optimization and Its Application to Online-Tuning PID Parametres in Idle Speed Engine Control System[C].Fukuoka International Congress Center, Japan, 2009. 
[10]Stefano Di Cairano. Model Predictive Idle Speed Control:Design,Analysis and Experimental Evaluation[J].IEEE TRANSACTIONS ON CONTROL SYSTEMS TECHNOLOGY, 2012:84-97.

[11]Zhang Jinlong, Zhao Fusheng. Gasoline engine idle speed of predictive control [J]. Journal of nanjing forestry university (natural science edition). 2002 (1) : $50 \sim 52$.

[12]Huiqing,Quyand,Minggao.Adaptive variable structure controllers and application to engine idle speed control simulation[J].Chinese Journal of Mechanical Engineering. 2003,16(2): 171 174

[13] ZHANG Y,KURIHARA N.A study of discrete-time sliding mode control[C].International conference on Industrial Technology.Hachinobe,Japan:IEEE,2011:27-32.

[14] Fan Chengde,Li Xiqin. Based on the theory of the LPG engine idle speed control study [J]. Journal of internal combustion engine engineering. 2007:58-62.

[15] Li Xiqin, Liu bing, Song Deyu. Robust control theory is applied to the CNG engine idle speed control [J]. Journal of automobile engineering. 56-59.

[16] Guo Hongzhi. Gasoline engine idle speed control method research [D], under the condition of jilin university, China, 2009 .

[17]Zhang Chiyun,Guo Linjia. Modern automobile engine electronic control fuel injection system research [J]. Journal of Shanghai university of engineering science, 2004, 17 (4) : $311 \sim 314$.

[18] I. Kawasumi, Y. Yasui, K. Higashitani and H. Ito. Cooperated Control of Multi Stage Injection System for Direct Injection Gasoline Engine[C].Proceedings of the 2006 American Control Conference Minneapolis, Minnesota, USA, June 14-16, 2006. 